\title{
Thinking outside the cavity: effusion lymphoma primary to bone marrow
}

Sean $\mathrm{Gu}^{1}$, Zenggang $\mathrm{Pan}^{2}$, and Mina $\mathrm{Xu}^{2}$

${ }^{1}$ Yale University School of Medicine

${ }^{2}$ Yale School of Medicine

January 6, 2021

\begin{abstract}
Primary effusion lymphoma (PEL) is a distinct disease entity of large B-cell lymphomas most often occurring in immunocompromised patients. We present a rare case of extracavitary PEL primary to the bone marrow in a HIV-positive patient.
\end{abstract}

Thinking outside the cavity: effusion lymphoma primary to bone marrow

Sean X. Gu${ }^{1}$, Zenggang $\mathrm{Pan}^{2}$, and Mina L. $\mathrm{Xu}^{1,2}$

${ }^{1}$ Department of Laboratory Medicine, Yale School of Medicine, New Haven, CT 06511

${ }^{2}$ Department of Pathology, Yale School of Medicine, New Haven, CT 06510

Corresponding author:

Sean X. Gu, MD, PhD

Clinical Fellow, Hematopathology

Department of Laboratory Medicine

Yale School of Medicine

55 Park St, New Haven, CT 06511

Email: sean.gu@yale.edu

Keywords: primary effusion lymphoma, extracavitary, AIDS-related lymphoma

Word count, text: 198

Word count, abstract: 36

Number of tables: 0

Number of figures: 1

Number of references: 2

Key Clinical Message:

This case illustrates a rare and aggressive entity in AIDS-related lymphoproliferative disorders and highlights the importance and challenges of recognizing PEL outside of cavitary lesions.

\section{ABSTRACT}


Primary effusion lymphoma (PEL) is a distinct disease entity of large B-cell lymphomas most often occurring in immunocompromised patients. We present a rare case of extracavitary PEL primary to the bone marrow in a HIV-positive patient.

\section{CASE DESCRIPTION}

A 41-year-old HIV-positive male presented to the emergency department with fever, malaise, and shortness of breath. Splenomegaly was noted on physical exam but no lymphadenopathy or cavitary effusions. His viral load was markedly elevated with CD4 count of 16 cells $/ \mu \mathrm{L}$, and he was pancytopenic. Clinical suspicion was high for infection, acute leukemia, or hemophagocytic lymphohistiocytosis. Bone marrow biopsy showed sheets of large atypical mononuclear cells with open chromatin, distinct nucleoli, and ample basophilic cytoplasm (Figures 1A \& B). Tumor cells were positive for CD138 (Figure 1C) and CD30 (Figure 1D) while negative for CD45, CD3, CD20, PAX5, OCT2, and CD79a. Differential diagnoses included plasmablastic lymphoma, plasma cell myeloma with plasmablastic features, and primary effusion lymphoma (PEL). Both EBV by in situ hybridization (Figure 1E) and HHV-8 (Figure 1F) were positive in tumor cells; hence, the diagnosis of extracavitary PEL was rendered. Chemotherapy with EPOCH was initiated; nevertheless, the patient died of disease 19 days after diagnosis.

PEL is a distinct clinicopathologic entity of AIDS-related lymphomas. Extracavitary PEL is a rare clinical variant of PEL that presents with solid tumor lesions in absence of malignant serous effusions. ${ }^{1,2}$ This case highlights the importance of recognizing PEL outside of cavitary lesions.

\section{CONFLICTS OF INTEREST}

None declared.

\section{AUTHOR CONTRIBUTIONS}

SXG collected patient information and wrote the manuscript. ZP provided support for photomicrographs and helped revise the manuscript. MLX provided insights and comments and helped revise the manuscript. All the authors approved the final version.

\section{ACKNOWLEDGMENTS}

None.

\section{REFERENCES}

1. Chadburn A, Hyjek E, Mathew S, Cesarman E, Said J, Knowles DM. KSHV-positive solid lymphomas represent an extra-cavitary variant of primary effusion lymphoma. Am J Surg Pathol . 2004;28(11):14011416.

2. Pan ZG, Zhang QY, Lu ZB, et al. Extracavitary KSHV-associated large B-Cell lymphoma: a distinct entity or a subtype of primary effusion lymphoma? Study of 9 cases and review of an additional 43 cases. $\mathrm{Am}$ J Surg Pathol . 2012;36(8):1129-1140. 


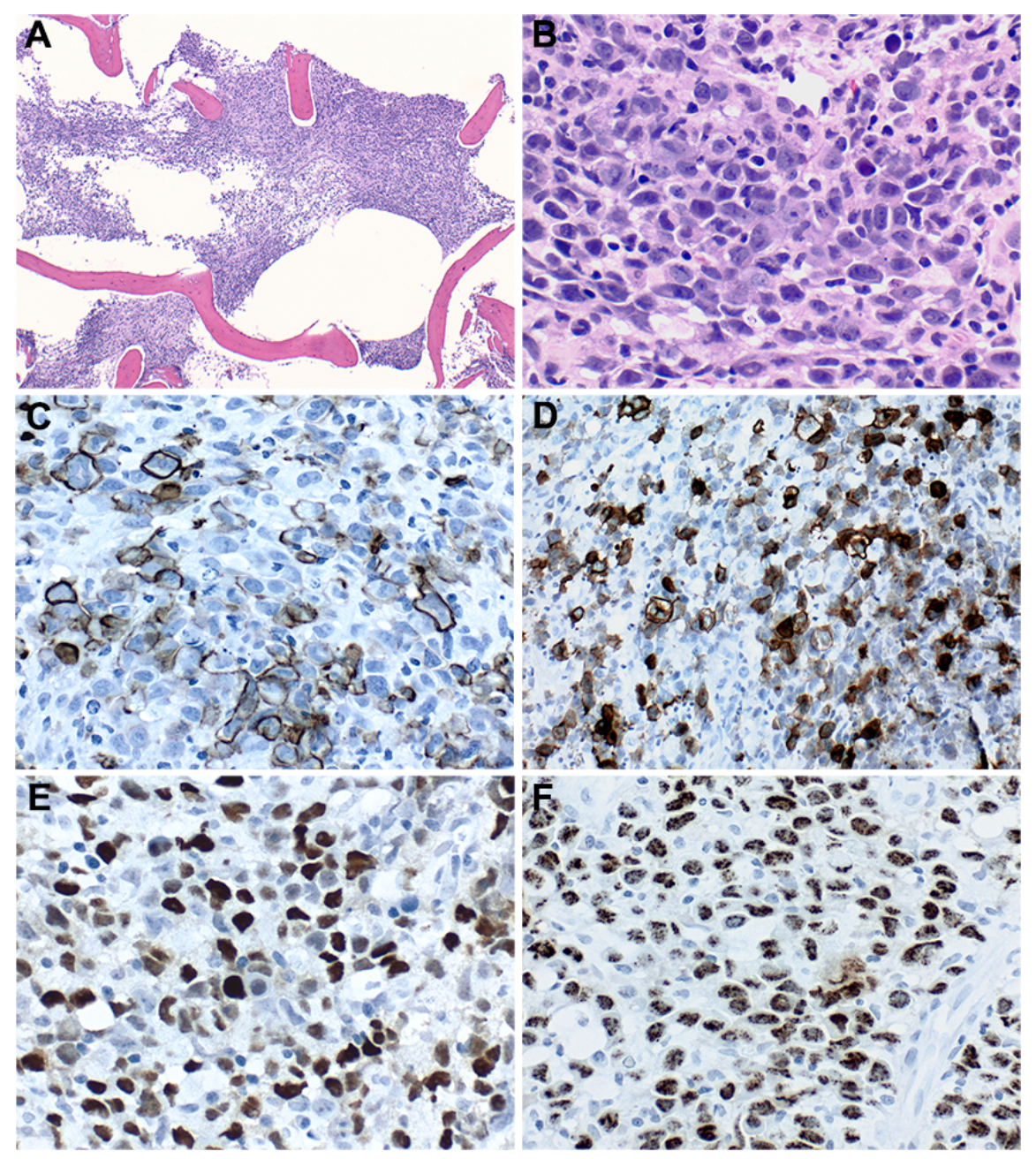

